NONCOMMUTATIVE GEOMETRY

AND QUANTUM GROUPS

BANACH CENTER PUBLICATIONS, VOLUME 61

INSTITUTE OF MATHEMATICS

POLISH ACADEMY OF SCIENCES

WARSZAWA 2003

\title{
K-THEORY OF BOUTET DE MONVEL'S ALGEBRA
}

\author{
SEVERINO T. MELO \\ Instituto de Matemática e Estatística, Universidade de São Paulo \\ Caixa Postal 66281, 05311-970 São Paulo, Brazil \\ E-mail:melo@ime.usp.br \\ RYSZARD NEST \\ Department of Mathematics, University of Copenhagen \\ Universitetsparken 5, 2100 Copenhagen, Denmark \\ E-mail: rnest@math.ku.dk \\ ELMAR SCHROHE \\ Institut für Mathematik, Universität Potsdam, Postfach 601553, 14415 Potsdam, Germany \\ E-mail: schrohe@math.uni-potsdam.de
}

\begin{abstract}
We consider the norm closure $\mathfrak{A}$ of the algebra of all operators of order and class zero in Boutet de Monvel's calculus on a compact manifold $X$ with boundary $\partial X$. Assuming that all connected components of $X$ have nonempty boundary, we show that $K_{1}(\mathfrak{A}) \simeq K_{1}(C(X)) \oplus$ ker $\chi$, where $\chi: K_{0}\left(C_{0}\left(T^{*} \dot{X}\right)\right) \rightarrow \mathbb{Z}$ is the topological index, $T^{*} \dot{X}$ denoting the cotangent bundle of the interior. Also $K_{0}(\mathfrak{A})$ is topologically determined. In case $\partial X$ has torsion free K-theory, we get $K_{0}(\mathfrak{A}) \simeq K_{0}(C(X)) \oplus K_{1}\left(C_{0}\left(T^{*} \dot{X}\right)\right)$.
\end{abstract}

Let $X$ be a compact $n$-dimensional manifold, with boundary $\partial X$ and interior $\dot{X}$, embedded in a closed manifold $\Omega$ of the same dimension. Let $E$ and $F$ be smooth complex vector bundles over $X$ and $\partial X$, respectively, $E$ being the restriction of a bundle $\tilde{E}$ over $\Omega$. Given a pseudodifferential operator $P$ on $\tilde{E}$, we denote by $P_{+} u$ the restriction to $\dot{X}$ of $P$ applied to the extension by zero of $u \in C^{\infty}(E)$ to $\Omega$. This defines a mapping from $C^{\infty}(E)$ to $C^{\infty}\left(\left.E\right|_{\dot{X}}\right)$, completely determined by the restriction of $P$ to $\dot{X}$. $P$ has the transmission property if the image of $P_{+}$is contained in $C^{\infty}(E)$.

We denote by $\mathcal{A}_{E F}$ the set of all polyhomogeneous Green operators

$$
A=\left(\begin{array}{cc}
P_{+}+G & K \\
T & S
\end{array}\right): \begin{array}{ccc}
C^{\infty}(E) & & C^{\infty}(E) \\
& C^{\infty}(F) & \\
& & C^{\infty}(F)
\end{array},
$$

2000 Mathematics Subject Classification: Primary 58J40, 46L80; Secondary 58J32, 35S15, $19 \mathrm{~K} 56$. 
of order and class zero, as defined by Boutet de Monvel [2]. In (1), $P$ is a pseudodifferential operator with the transmission property, $S$ is a pseudodifferential operator on $F$. $G, K$, and $T$ are regularizing in the interior of $X$ and locally at the boundary are given as pseudodifferential operators on $\mathbb{R}^{n-1}$ with operator-valued symbols. They are called, respectively, singular Green operators, Poisson operators and trace operators. Detailed expositions of Boutet de Monvel's calculus can be found in $[4,9]$. We adopt here notation and definitions of [4].

We write $\mathfrak{L}(\mathfrak{H})$ for the set of all bounded operators on the Hilbert space $\mathfrak{H}=L^{2}(E) \oplus$ $H^{-\frac{1}{2}}(F), H^{-\frac{1}{2}}$ denoting the usual Sobolev space. $\mathcal{A}_{E F}$ is an adjoint-invariant sub-algebra of $\mathfrak{L}(\mathfrak{H})$, closed under holomorphic functional calculus [10]. It then has the same K-theory as its norm closure, which we denote by $\mathfrak{A}_{E F}$.

Assuming that the rank of $E$ is nonzero (the case $E=0$ belongs to classical index theory on closed manifolds), it follows from the rules of Boutet de Monvel's calculus that $\mathfrak{A}_{E F}$ is strongly Morita equivalent to the corresponding algebra for the case when $E$ and $F$ are rank-one trivial bundles. Since strongly Morita equivalent $\mathrm{C}^{*}$-algebras have the same K-theory [3], we now assume that $E=X \times \mathbb{C}$ and $F=\partial X \times \mathbb{C}$, and write $\mathcal{A}$ and $\mathfrak{A}$ instead of $\mathcal{A}_{E F}$ and $\mathfrak{A}_{E F}$. In this paper we compute the K-groups of $\mathfrak{A}$, pointing out relations between this computation and Boutet de Monvel's index theorem. Proofs are only sketched, details given in [8].

Our main tool is the analysis of the boundary principal symbol, *-homomorphism defined by Boutet de Monvel on $\mathcal{A}$ already in [2]. In order to describe it, one needs to use coordinates. Let the intersection, if not empty, of $\partial X$ with the domain of any chart be given by $x_{n}=0$, and let $x^{\prime}=\left(x_{1}, \cdots, x_{n-1}\right)$. With respect to such a chart, the operators $G, K$ and $T$ are pseudodifferential operators on $\mathbb{R}^{n-1}$, with operator-valued symbols $g\left(x^{\prime}, \xi^{\prime}, D_{n}\right): \mathcal{S}\left(\overline{\mathbb{R}}_{+}\right) \rightarrow \mathcal{S}\left(\overline{\mathbb{R}}_{+}\right), k\left(x^{\prime}, \xi^{\prime}, D_{n}\right): \mathbb{C} \rightarrow \mathcal{S}\left(\overline{\mathbb{R}}_{+}\right)$, and $t\left(x^{\prime}, \xi^{\prime}, D_{n}\right): \mathcal{S}\left(\overline{\mathbb{R}}_{+}\right) \rightarrow \mathbb{C} ;$ where $\mathcal{S}\left(\overline{\mathbb{R}}_{+}\right)$denotes the space of restrictions to $\overline{\mathbb{R}}_{+}=\left\{x_{n} ; x_{n} \geq 0\right\}$ of functions in the Schwartz class $\mathcal{S}(\mathbb{R})$. These symbols, in their turn, are defined by complex-valued smooth functions $g\left(x^{\prime}, \xi^{\prime}, \xi_{n}, \eta_{n}\right), k\left(x^{\prime}, \xi^{\prime}, \xi_{n}\right)$, and $t\left(x^{\prime}, \xi^{\prime}, \xi_{n}\right)$, also called symbols, even though our operators are not usual pseudodifferential operators in the last variable $x_{n}$. In fact, for each $\left(x^{\prime}, \xi^{\prime}\right) \in \mathbb{R}^{n-1} \times \mathbb{R}^{n-1}, g\left(x^{\prime}, \xi^{\prime}, D_{n}\right), k\left(x^{\prime}, \xi^{\prime}, D_{n}\right)$, and $t\left(x^{\prime}, \xi^{\prime}, D_{n}\right)$ are integral operators with kernels $(2 \pi)^{-2} \hat{g}\left(x^{\prime},-x_{n}, y_{n}, \xi^{\prime}\right),(2 \pi)^{-1} \hat{k}\left(x^{\prime},-x_{n}, \xi^{\prime}\right)$ and $(2 \pi)^{-1} \hat{t}\left(x^{\prime}, y_{n}, \xi^{\prime}\right)$, respectively, where $\hat{g}\left(x^{\prime}, \cdot, \cdot, \xi^{\prime}\right), \hat{k}\left(x^{\prime}, \cdot, \xi^{\prime}\right)$ and $\hat{t}\left(x^{\prime}, \cdot, \xi^{\prime}\right)$ denote the Fourier transforms of $g\left(x^{\prime}, \xi^{\prime}, \cdot, \cdot\right), k\left(x^{\prime}, \xi^{\prime}, \cdot\right)$ and $t\left(x^{\prime}, \xi^{\prime}, \cdot\right)$ (see [4], (2.3.25) and (2.4.4) to (2.4.7)). This explains why the extra covariable $\eta_{n}$ is needed for $g$. The symbol classes to which $g, k$, and $t$ belong are such that, for each $\left(x^{\prime}, \xi^{\prime}\right)$, the kernels of $k\left(x^{\prime}, \xi^{\prime}, D_{n}\right)$ and $t\left(x^{\prime}, \xi^{\prime}, D_{n}\right)$ belong to $\mathcal{S}\left(\overline{\mathbb{R}}_{+}\right)$, while that of $g\left(x^{\prime}, \xi^{\prime}, D_{n}\right)$ is the restriction to $\overline{\mathbb{R}}_{+} \times \overline{\mathbb{R}}_{+}$of a function in $\mathcal{S}\left(\mathbb{R}^{2}\right)$; in particular, $g\left(x^{\prime}, \xi^{\prime}, D_{n}\right)$ is a compact operator on $L^{2}\left(\overline{\mathbb{R}}_{+}\right)$.

The polyhomogeneity hypothesis means that the local symbols $p\left(x^{\prime}, x_{n}, \xi^{\prime}, \xi_{n}\right)$, $g\left(x^{\prime}, \xi^{\prime}, \xi_{n}, \eta_{n}\right), k\left(x^{\prime}, \xi^{\prime}, \xi_{n}\right), t\left(x^{\prime}, \xi^{\prime}, \xi_{n}\right)$, and $s\left(x^{\prime}, \xi^{\prime}\right)$ for $P, G, K, T$, and $S$ possess asymptotic expansions in homogeneous components. Let $p_{0}, g_{0}, k_{0}, t_{0}$, and $s_{0}$ denote the leading terms in those expansions. The boundary principal symbol $\gamma(A)$ of the Green operator $A \in \mathcal{A}$ is a function defined on the cosphere bundle of the boundary, $S^{*} \partial X$, valued in Green operators on $\overline{\mathbb{R}}_{+}$. For each $\left(x^{\prime}, \xi^{\prime}\right), \gamma(A)\left(x^{\prime}, \xi^{\prime}\right)$ is obtained from the symbols (regarded as functions of $\xi_{n}$ and $\left.\eta_{n}\right) p_{0}\left(x^{\prime}, 0, \xi^{\prime}, \xi_{n}\right), g_{0}\left(x^{\prime}, \xi^{\prime}, \xi_{n}, \eta_{n}\right), k_{0}\left(x^{\prime}, \xi^{\prime}, \xi_{n}\right)$, 
$t_{0}\left(x^{\prime}, \xi^{\prime}, \xi_{n}\right)$, and $s_{0}\left(x^{\prime}, \xi^{\prime}\right)$. That is, we have:

$$
\gamma(A)\left(x^{\prime}, \xi^{\prime}\right)=\left(\begin{array}{cc}
p_{0}\left(x^{\prime}, 0, \xi^{\prime}, D_{n}\right)_{+}+g_{0}\left(x^{\prime}, \xi^{\prime}, D_{n}\right) & k_{0}\left(x^{\prime}, \xi^{\prime}, D_{n}\right) \\
t_{0}\left(x^{\prime}, \xi^{\prime}, D_{n}\right) & s_{0}\left(x^{\prime}, \xi^{\prime}\right)
\end{array}\right) .
$$

This is invariantly defined ([9], 2.3.3.1, Theorem 3; [4], Theorem 2.4.11), if we restrict ourselves to an atlas whose changes of variables among boundary charts preserve the variable $x_{n}$, not only the set $\left\{x_{n}=0\right\}$. In other words, while the algebra depends only on the manifold, the bondary principal symbol depends on the choice of an atlas.

The principal symbol of a Green operator is, by definition, the principal symbol of $P$, $p_{0}(x, \xi)$, regarded as a function on the cosphere bundle $S^{*} X$. We denote this by $\sigma(A)=$ $\sigma(P)$. Invertibility of the two symbols $\sigma(A)$ and $\gamma(A)$ is equivalent to the Fredholm property for a Green operator $A$ ([2], Theorem 5.1). That also follows from the estimate $[5,9]$

$$
\inf _{C \in \mathfrak{K}}\|A+C\|=\max \{\|\sigma(A)\|,\|\gamma(A)\|\}, \text { for all } A \in \mathcal{A},
$$

which generalizes the classical estimate for the $L^{2}$-norm, modulo compacts, of a pseudodifferential operator on a closed manifold (then, $\gamma=0$ ). We have denoted by $\mathfrak{K} \subset \mathfrak{L}(\mathfrak{H})$ the compact ideal, by $\|\sigma(A)\|$ the supremum norm of $\sigma(A)$ on $S^{*} X$, and by $\|\gamma(A)\|$ the supremum of $\left\|\gamma(A)\left(x^{\prime}, \xi^{\prime}\right)\right\|_{\mathfrak{L}\left(L^{2}\left(\overline{\mathbb{R}}_{+}\right) \oplus \mathbb{C}\right)}$ over all $\left(x^{\prime}, \xi^{\prime}\right)$ in $S^{*} \partial X$. It follows from (2), in particular, that $\gamma$ extends to the $\mathrm{C}^{*}$-algebra homomorphism

$$
\bar{\gamma}: \mathfrak{A} \rightarrow C\left(S^{*} \partial X\right) \otimes \mathfrak{L}\left(L^{2}\left(\overline{\mathbb{R}}_{+}\right) \oplus \mathbb{C}\right) .
$$

There is a close connection between Boutet de Monvel's calculus and the theory of Toeplitz operators, as we now explain. Let $U: L^{2}\left(\mathbb{S}^{1}\right) \rightarrow L^{2}(\mathbb{R})$ denote the unitary mapping $U g(t)=\frac{\sqrt{2}}{1+i t} g\left(\frac{1-i t}{1+i t}\right), \mathcal{H}_{-1}$ denote the image of $C^{\infty}\left(\mathbb{S}^{1}\right)$ under $U$, and $\mathcal{H}_{0}=\mathcal{H}_{-1} \oplus \mathbb{C}$. The image by $U$ of the Hardy space $H^{2}$ is equal to $F\left(L^{2}\left(\overline{\mathbb{R}}_{+}\right)\right)$, with $F$ denoting the Fourier transform on $\mathbb{R}$. Given $p \in \mathcal{H}_{0}$, the truncated Fourier multiplier $p(D)_{+}$equals $F^{-1} U T_{\tilde{p}} U^{-1} F$, with $T_{\tilde{p}}$ denoting the Toeplitz operator of symbol $\tilde{p}(z)=p\left(\frac{i z-i}{z+1}\right)$. Denoting also by $\mathfrak{K}$ the compact ideal on $L^{2}\left(\overline{\mathbb{R}}_{+}\right)$, one then gets (see also [4], Lemma 3.1.5):

LEMma 1. $\inf _{C \in \mathfrak{K}}|| p(D)_{+}+C\|=\| p(D)_{+} \|=\sup |p|$, for all $p \in \mathcal{H}_{0}$.

Next we introduce notation needed to describe the kernel and the image of $\bar{\gamma}$. Those descriptions will then be used to compute the $K$-groups of $\mathfrak{A} / \mathfrak{K}$. That will be the central part of this paper, in view of the following remark.

REMARK 2. For sufficiently large $k$, there exist matrices in $M_{k}(\mathfrak{A})$ which, regarded as operators on $\mathfrak{H}^{k}$, have Fredholm index one. Hence, $K_{0}(\mathfrak{A})$ is isomorphic to $K_{0}(\mathfrak{A} / \mathfrak{K})$, and $K_{1}(\mathfrak{A})$ is isomorphic to the kernel of the Fredholm index, regarded as a mapping from $K_{1}(\mathfrak{A} / \mathfrak{K})$ to $K_{0}(\mathfrak{K}) \simeq \mathbb{Z}$.

Let $m$ denote the mapping $C(X) \ni f \mapsto\left(\begin{array}{cc}f & 0 \\ 0 & \left.f\right|_{\partial X}\end{array}\right) \in \mathfrak{A}$ (we use the same symbol to denote a function and the corresponding multiplication operator). We define $b: C(\partial X) \rightarrow \operatorname{Im} \bar{\gamma}$ by $b\left(\left.f\right|_{\partial X}\right)=\bar{\gamma}(m(f))$. Let $\mathfrak{W}$ denote the $\mathrm{C}^{*}$-subalgebra of $\mathfrak{L}\left(L^{2}\left(\overline{\mathbb{R}}_{+}\right) \oplus \mathbb{C}\right)$ generated by all operators whose upper left corner is of the form $p(D)_{+}$, 
$p \in \mathcal{H}_{0}$. If $p(x, \xi)$ is the symbol of a pseudodifferential operator with the transmission property, then $p\left(x^{\prime}, 0, \xi^{\prime}, \cdot\right) \in \mathcal{H}_{0}$, for all $\left(x^{\prime}, \xi^{\prime}\right)$. The range of $\bar{\gamma}, \operatorname{Im} \bar{\gamma}$, is therefore contained in $C\left(S^{*} \partial X\right) \otimes \mathfrak{W}$. Let $\mathfrak{W}_{0}$ denote the kernel of the *-homomorphism $\mathfrak{W} \ni\left(\begin{array}{cc}p(D)_{+} & \cdot \\ \cdot & \cdot\end{array}\right) \mapsto p(\infty) \in \mathbb{C}$.

Theorem 3. $\operatorname{Im} \bar{\gamma}=\left(C\left(S^{*} \partial X\right) \otimes \mathfrak{W}_{0}\right) \oplus \operatorname{Im} b \simeq\left(C\left(S^{*} \partial X\right) \otimes \mathfrak{W}_{0}\right) \oplus C(\partial X)$

Proof. For each $f \in L^{2}\left(\overline{\mathbb{R}}_{+}\right)$, let us denote by $\langle f|$ the linear functional $\psi \mapsto \int f \psi$; and by $|f\rangle$ the linear map, from $\mathbb{C}$ to $L^{2}\left(\overline{\mathbb{R}}_{+}\right)$, of multiplication by $f$. Let there be given $\varphi \in C^{\infty}\left(S^{*} X\right)$ with small support; and arbitrary $f_{11} \in C_{c}^{\infty}(\mathbb{R}), f_{12}, f_{21} \in C_{c}^{\infty}\left(\overline{\mathbb{R}}_{+}\right)$and $s \in \mathbb{R}$. One can explicitly construct a Green operator whose boundary principal symbol is, roughly speaking, "equal" to $\varphi \otimes\left(\begin{array}{cc}f_{11}(D)_{+} & \left|f_{12}\right\rangle \\ \left\langle f_{21}\right| & s\end{array}\right)$. A density argument then gives $C\left(S^{*} \partial X\right) \otimes \mathfrak{W}_{0} \subset \operatorname{Im} \bar{\gamma}$

We will be finished if we show that the intersection of the upper left corner of $\operatorname{Im} \bar{\gamma}$ and $C\left(S^{*} \partial X\right) \otimes(\mathbb{C} \cdot I)$ is contained in $C(\partial X) \otimes(\mathbb{C} \cdot I)$, where $I$ denotes the identity on $L^{2}\left(\overline{\mathbb{R}}_{+}\right)$. Regarding the mapping $A \mapsto\left(\begin{array}{cc}A & 0 \\ 0 & 0\end{array}\right)$ as an inclusion of the upper left corner as a subalgebra of $\mathfrak{A}$, and analogously for $\operatorname{Im} \bar{\gamma}$, let $f \in C\left(S^{*} \partial X\right)$ be so that $f \otimes I \in \operatorname{Im} \bar{\gamma}$. Then, for every $\delta>0$, there exist a zero-order polyhomogeneous pseudodifferential operator with the transmission property $P$ and a singular Green operator $G$ such that $\left\|\gamma\left(P_{+}\right)\left(x^{\prime}, \xi^{\prime}\right)-\gamma(G)\left(x^{\prime}, \xi^{\prime}\right)-f\left(x^{\prime}, \xi^{\prime}\right) I\right\|<\delta$, for all $\left(x^{\prime}, \xi^{\prime}\right) \in S^{*} \partial X$. For each $\left(x^{\prime}, \xi^{\prime}\right) \in S^{*} \partial X$, since $\gamma(G)\left(x^{\prime}, \xi^{\prime}\right)$ is compact, we get:

$$
\inf _{C \in \mathfrak{K}}\left\|\gamma\left(P_{+}\right)\left(x^{\prime}, \xi^{\prime}\right)-f\left(x^{\prime}, \xi^{\prime}\right) I+C\right\|<\delta .
$$

Lemma 1 implies that the left-hand side of the above inequality is equal to

$$
\sup _{\xi_{n} \in \mathbb{R}}\left|p_{0}\left(x^{\prime}, 0, \xi^{\prime}, \xi_{n}\right)-f\left(x^{\prime}, \xi^{\prime}\right)\right|=\sup _{\xi_{n} \neq 0}\left|p_{0}\left(x^{\prime}, 0, \frac{\xi^{\prime}}{\left|\xi_{n}\right|}, \pm 1\right)-f\left(x^{\prime}, \xi^{\prime}\right)\right|,
$$

which is greater then or equal to $\left|p_{0}\left(x^{\prime}, 0,0,+1\right)-f\left(x^{\prime}, \xi^{\prime}\right)\right|$, with $p_{0}$ denoting the (homogeneous) principal symbol of $P$.

The proof of the following theorem uses the already mentioned norm estimates for Toeplitz operators and for pseudodifferential operators on closed manifolds. It also depends on analogous results for Green operators: the estimate (2) for the norm of the quotient $\mathfrak{A} / \mathfrak{K}$, and the norm estimate, essentially given by $(3)$, for the quotient of $\mathfrak{A}$ by an ideal $\mathfrak{I} \supsetneq \mathfrak{K}$.

THEOREM 4. The principal symbol induces an isomorphism from $\mathrm{ker} \bar{\gamma} / \mathfrak{K}$ to the algebra $C_{0}\left(S^{*} \dot{X}\right)$ of all continuous functions on $S^{*} X$ which vanish over $\partial X$.

Proof. Let $\mathfrak{I}$ denote the $\mathrm{C}^{*}$-subalgebra of $\mathfrak{L}(\mathfrak{H})$ generated by $\mathfrak{K}$ and all Green operators of the form $\varphi Q \psi$, with $Q$ a zero-order polyhomogeneous pseudodifferential operator on $\dot{X}$ and $\varphi, \psi \in C_{c}^{\infty}(\dot{X})$ (we are again regarding the upper left corner as a subalgebra). The estimate

$$
\inf _{C \in \mathfrak{K}}\|\varphi Q \psi+C\|=\sup _{S^{*} \dot{X}}|\varphi \psi \sigma(Q)|
$$


follows from [7], Theorem A.4, or from [6], Theorem 3.3. This gives $\mathfrak{I} / \mathfrak{K} \simeq C_{0}\left(S^{*} \dot{X}\right)$. Since $\operatorname{ker} \gamma \subset \mathfrak{I}$, and $\operatorname{ker} \gamma$ contains a dense subset of $\mathfrak{I}$, we have $\mathfrak{I}=\overline{\operatorname{ker} \gamma} \subseteq \operatorname{ker} \bar{\gamma}$. To prove the reverse inclusion, it is enough to show that

$$
\inf _{\varphi, \psi, Q}\left\|P_{+}+G+\varphi Q \psi\right\| \leq c\left\|\gamma\left(P_{+}+G\right)\right\|,
$$

for all $P_{+}+G$ in the upper left corner of $\mathcal{A}$. We give a hint of how that can be proven, loosely using local coordinates.

Let $\tilde{P}$ denote the pseudodifferential operator of symbol $p\left(x^{\prime}, 0, \xi^{\prime}, \xi_{n}\right)$, where $p$ is the symbol of $P$. Since $\gamma(\tilde{P})=\gamma(P), P-\tilde{P} \in \mathfrak{I}$. Moreover, there exists a compact $C$ such that $\|\tilde{P}+C\| \leq 2 \sup _{x^{\prime}, \xi^{\prime}, \xi_{n}}\left|p_{0}\left(x^{\prime}, 0, \xi^{\prime}, \xi_{n}\right)\right|$. By Lemma 1 , for each $\left(x^{\prime}, \xi^{\prime}\right)$,

$$
\sup _{\xi_{n}}\left|p_{0}\left(x^{\prime}, 0, \xi^{\prime}, \xi_{n}\right)\right|=\left\|p_{0}\left(x^{\prime}, 0, \xi^{\prime}, D_{n}\right)_{+}\right\|=\left\|\gamma\left(P_{+}\right)\left(x^{\prime}, \xi^{\prime}\right)\right\|
$$

This proves (3) when $G=0$. For $P=0,(3)$ follows from (2). The general case follows then from Lemma 1 , using that $\gamma(G)\left(x^{\prime}, \xi^{\prime}\right)$ is compact for each $\left(x^{\prime}, \xi^{\prime}\right)$.

Since $\operatorname{Im} \bar{\gamma}$ and $\mathfrak{A} / \mathfrak{I}$ are isomorphic, and $K_{*}\left(C\left(S^{*} \partial X\right) \otimes \mathfrak{W}_{0}\right)$ vanishes, we get:

Corollary $5 . b_{*}: K_{*}(C(\partial X)) \rightarrow K_{*}(\mathfrak{A} / \mathfrak{I})$ is an isomorphism.

Now we consider the exact sequence of $\mathrm{C}^{*}$-algebras

$$
0 \rightarrow C_{0}\left(T^{*} \dot{X}\right) \rightarrow C_{0}\left(B^{*} \dot{X}\right) \rightarrow C_{0}\left(S^{*} \dot{X}\right) \rightarrow 0
$$

induced by the restriction mapping, each cosphere being regarded as the infinite points of the radial compactification of the corresponding cotangent space. Since the bundle of closed balls $B^{*} \dot{X}$ is homotopically equivalent to $\dot{X},(4)$ yields the cyclic exact sequence of abelian groups (see [1], 9.3.1)

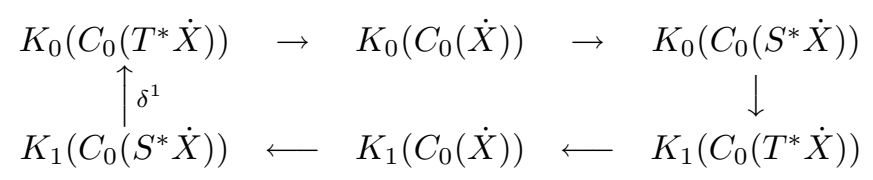

If one assumes that every connected component of $X$ has nonempty boundary, then any finite number of zeroes of a section of $T^{*} X$ may be continuously deformed to $\Omega \backslash X$. This implies that $S^{*} \dot{X}$ possesses a continuous section $\Sigma$. Composition with $\Sigma$ defines a $\mathrm{C}^{*}$-algebra homomorphism $s: C_{0}\left(S^{*} \dot{X}\right) \rightarrow C_{0}(\dot{X})$ such that $s \circ m^{\prime}$ is the identity, with $m^{\prime}: C_{0}(\dot{X}) \rightarrow C_{0}\left(S^{*} \dot{X}\right)$ denoting the pullback under the bundle projection. Since the upper-right and the lower-left arrows in (5) are homomorphisms induced by $m^{\prime},(5)$ becomes the two split exact sequences

$$
0 \rightarrow K_{i}\left(C_{0}(\dot{X})\right) \stackrel{m_{*}^{\prime}}{\underset{s_{*}}{\longrightarrow}} K_{i}\left(C_{0}\left(S^{*} \dot{X}\right)\right) \rightarrow K_{1-i}\left(C_{0}\left(T^{*} \dot{X}\right)\right) \rightarrow 0
$$

$i=0,1$. In particular, $K_{i}\left(C_{0}\left(S^{*} \dot{X}\right)\right)$ is isomorphic to the direct sum $K_{i}\left(C_{0}(\dot{X})\right) \oplus$ $K_{1-i}\left(C_{0}\left(T^{*} \dot{X}\right)\right)$. With respect to this isomorphism, for each $i$, the canonical projection of $K_{i}\left(C_{0}\left(S^{*} \dot{X}\right)\right)$ onto $K_{1-i}\left(C_{0}\left(T^{*} \dot{X}\right)\right)$ corresponds to a connecting mapping in (5), while the injection of $K_{i}\left(C_{0}(\dot{X})\right)$ into $K_{i}\left(C_{0}\left(S^{*} \dot{X}\right)\right)$ is the group homomorphism induced by $m^{\prime}$. 
Defining $\tilde{m}$ by $C(X) \ni f \mapsto \tilde{m}(f)=[m(f)] \in \mathfrak{A} / \mathfrak{K}$, with [·] denoting the canonical projection from $\mathfrak{A}$ onto $\mathfrak{A} / \mathfrak{K}$, we obtain the commutative diagram

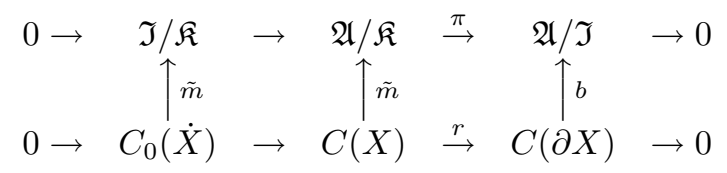

Under the isomorphism of Theorem 4 , the restriction of $\tilde{m}$ to $C_{0}(\dot{X})$ corresponds to $m^{\prime}$. Hence, the connecting mappings associated to the two exact sequences in (6) are the same, modulo the isomorphisms $b_{*}$ and the split injections $m_{*}^{\prime}$; i.e.,

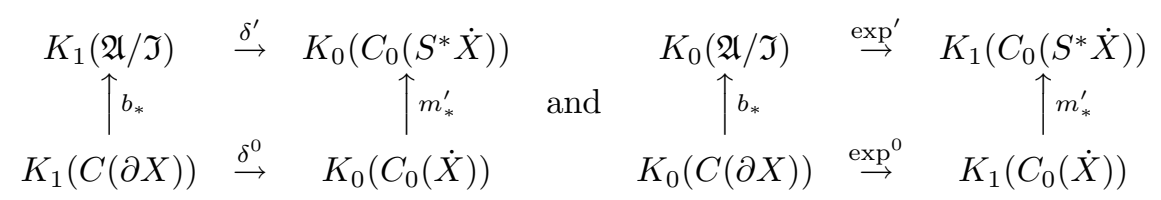

commute, with $\delta^{0}$ and $\exp ^{0}$ denoting the connecting mappings for the six-term exact sequence associated to the lower sequence in (6), and $\delta^{\prime}$ and $\exp ^{\prime}$ those for the upper one (modulo the isomorphism of Theorem 4). This implies:

THEOREM 6. If the boundary of every connected component is not empty, then, for each $i=0,1$, there is an exact sequence

$$
0 \rightarrow \operatorname{ker} r_{*}^{i} \oplus K_{1-i}\left(C_{0}\left(T^{*} \dot{X}\right)\right) \rightarrow K_{i}(\mathfrak{A} / \mathfrak{K}) \rightarrow \operatorname{Im} r_{*}^{i} \rightarrow 0
$$

where $r_{*}^{i}: K_{i}(C(X)) \rightarrow K_{i}(C(\partial X))$ is the homomorphism induced by the restriction to the boundary $r: C(X) \rightarrow C(\partial X)$.

Corollary 7. If, moreover, the $K$-groups of $C(\partial X)$ are torsion free, then $K_{i}(\mathfrak{A} / \mathfrak{K})$ is isomorphic to $K_{i}(C(X)) \oplus K_{1-i}\left(C_{0}\left(T^{*} \dot{X}\right)\right), i=0,1$.

Proof. Twice apply the fact that, if a sequence $0 \rightarrow A \rightarrow B \rightarrow C \rightarrow 0$ of abelian groups is exact and $C$ is free, then $B$ is isomorphic to $A \oplus C$.

EXAmple 8. Suppose that $X$, in addition to being compact and all its components having nonempty boundary, is orientable and has dimension two. Corollary 7 then implies that $K_{0}(\mathfrak{A} / \mathfrak{K}) \simeq K_{1}(\mathfrak{A} / \mathfrak{K}) \simeq \mathbb{Z}^{2 g+m}$, where $g$ denotes the genus of $X$ and $m$ the number of connected components of its boundary. By Remark 2 , we get $K_{0}(\mathfrak{A}) \simeq K_{0}(\mathcal{A}) \simeq \mathbb{Z}^{2 g+m}$ and $K_{1}(\mathfrak{A}) \simeq K_{1}(\mathcal{A}) \simeq \mathbb{Z}^{2 g+m-1}$.

Consequences of Boutet de Monvel's index theorem. A central point in Boutet de Monvel's paper is the definition of a homomorphism from $K_{1}(\mathfrak{A} / \mathfrak{K})$ to $K\left(T^{*} \dot{X}\right)$ ([2], Theorem 5.21; see also [9], 3.2.2.4, Theorem 1). Let us denote the composition of the canonical isomorphism $K_{0}\left(C_{0}\left(T^{*} \dot{X}\right)\right) \simeq K\left(T^{*} \dot{X}\right)$ with that homomorphism by ind : $K_{1}(\mathfrak{A} / \mathfrak{K}) \rightarrow K_{0}\left(C_{0}\left(T^{*} \dot{X}\right)\right.$ ) (we refer to [1], Section 1, for topological K-theory definitions and notation). Here we shall use how ind is defined, and how it relates to the Fredholm index, to give a more precise description of the structure of $K_{1}(\mathfrak{A} / \mathfrak{K})$,

Any $x \in K_{1}(\mathfrak{A} / \mathfrak{K})$ is of the form $x=[[A]]$, for some Fredholm $A \in M_{k}(\mathcal{A}),[[A]]$ denoting the $K_{1}$-class of the projection $[A]$ of $A$ onto $M_{k}(\mathfrak{A} / \mathfrak{K})$. If $A$ happens to be a bundle isomorphism near the boundary, $\sigma(A)$ is independent of the covariable near 
the boundary, and hence induces an element $d(\sigma(A))$ of $K\left(B^{*} X,\left.S^{*} X \cup T^{*} X\right|_{\partial X}\right)$. By definition, ind $(x)$ is the element of $K_{0}\left(C_{0}\left(T^{*} \dot{X}\right)\right)$ identified with $d(\sigma(A))$ via the isomorphisms $K_{0}\left(C_{0}\left(T^{*} \dot{X}\right)\right) \simeq K\left(T^{*} \dot{X}\right) \simeq K\left(B^{*} X,\left.S^{*} X \cup T^{*} X\right|_{\partial X}\right)$. Consequently we see that, if $x=[[A]] \in K_{1}(\mathfrak{I} / \mathfrak{K})$ is induced by $A \in M_{k}(\mathfrak{I} \oplus \mathbb{C})$, then ind $(x)$ corresponds, via the isomorphism $K_{1}(\mathfrak{I} / \mathfrak{K}) \simeq K_{1}\left(C_{0}\left(S^{*} \dot{X}\right)\right)$, to $\delta^{1}(x)$, where $\delta^{1}$ denotes the index mapping associated to (4). In other words: We have ind $\circ i_{*}=\delta^{1}$, for the homomorphism $i_{*}: K_{1}(\mathfrak{I} / \mathfrak{K}) \rightarrow K_{1}(\mathfrak{A} / \mathfrak{K})$ induced by the inclusion $i$ of $\mathfrak{I} / \mathfrak{K}$ into $\mathfrak{A} / \mathfrak{K}$. Moreover, since the principal symbol of $m(f)$, for any $f \in C(X)$, is an isomorphism over all $B^{*} X$, ind $\circ \tilde{m}_{*}=0$.

Using, moreover, the isomorphism $K_{1}\left(C_{0}\left(S^{*} \dot{X}\right)\right) \simeq K_{1}\left(C_{0}(\dot{X})\right) \oplus K_{0}\left(C_{0}\left(T^{*} \dot{X}\right)\right)$, we then obtain from (6) the commutative diagram

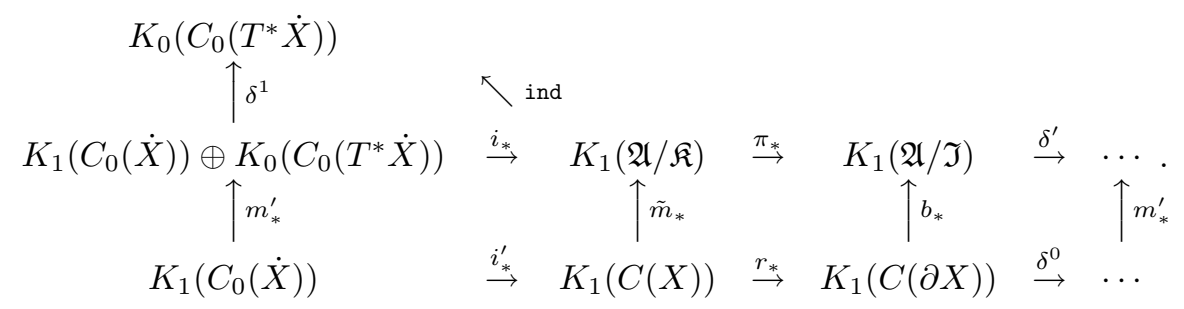

Some diagram chasing gives:

THEOREM 9. If $X$ is connected and $\partial X$ is not empty, then $K_{1}(\mathfrak{A} / \mathfrak{K})$ is isomorphic to $K_{1}(C(X)) \oplus K_{0}\left(C_{0}\left(T^{*} \dot{X}\right)\right)$. More precisely, in the above diagram, $\tilde{m}_{*}$ and the restriction of $i_{*}$ to $K_{0}\left(C_{0}\left(T^{*} \dot{X}\right)\right)$ are injective, and $K_{1}(\mathfrak{A} / \mathfrak{K})=\tilde{m}_{*}\left(K_{1}(C(X)) \oplus i_{*}\left(K_{0}\left(C_{0}\left(T^{*} \dot{X}\right)\right)\right)\right.$.

Even though the restriction of $i_{*}: K_{1}\left(C_{0}\left(S^{*} \dot{X}\right)\right) \rightarrow K_{1}(\mathfrak{A} / \mathfrak{K})$ to $K_{0}\left(C_{0}\left(T^{*} \dot{X}\right)\right)$ is injective, $i_{*}$ itself is not: For the case considered in Example 8, we get $\operatorname{ker} i_{*} \simeq \mathbb{Z}^{m-1}$.

COROLlary 10. With respect to the isomorphism of Theorem 9, ind corresponds to the canonical projection from $K_{1}(C(X)) \oplus K_{0}\left(C_{0}\left(T^{*} \dot{X}\right)\right)$ onto $K_{0}\left(C_{0}\left(T^{*} \dot{X}\right)\right)$.

The Fredholm index on $K_{1}(\mathfrak{A} / \mathfrak{K})$ is the composition of the topological index $\chi$ : $K_{0}\left(C_{0}\left(T^{*} \dot{X}\right)\right) \rightarrow \mathbb{Z}$ with ind ([2], Section 5.8; [9], 3.2.2.3, 3.2.2.4). By Remark 2, we then get:

Corollary 11. $K_{1}(\mathfrak{A})$ and $K_{1}(\mathcal{A})$ are isomorphic to $K_{1}(C(X)) \oplus \operatorname{ker} \chi$.

Below we denote by the same symbols matrices of continuous functions and the multiplication operators they define.

Corollary 12. If $A \in M_{k}(\mathfrak{A})$ defines a Fredholm operator on $\mathfrak{H}^{k}$, then, for some $l \geq k$, there exist: an l-by-l matrix $P$ of zero-order polyhomogeneous pseudodifferential operators on $\dot{X}$, an invertible $f \in M_{l}(C(X)), \varphi$ and $\psi \in C_{c}^{\infty}(\dot{X})$, and a continuous path of Fredholm operators in $M_{l}(\mathfrak{A})$ connecting $A \oplus 1_{l-k}$ to $A_{1} A_{2}$, where $A_{1}=\left(\begin{array}{cc}\varphi P \psi+I & 0 \\ 0 & I\end{array}\right)$ and $A_{2}=\left(\begin{array}{cc}f & 0 \\ 0 & \left.f\right|_{\partial X}\end{array}\right), I$ denoting both the identity on $L^{2}\left(X ; \mathbb{C}^{l}\right)$ and on $H^{-\frac{1}{2}}\left(\partial X ; \mathbb{C}^{l}\right)$.

Proof. This follows immediately from Theorem 9 and the definitions, after one notes that: $(i)$ the set of all such $A_{1}$ and the compact operators generate a dense subalgebra of 
$M_{l}(\mathfrak{I} \oplus \mathbb{C})$, and $(i i)$ any homotopy of invertibles in $M_{l}(\mathfrak{A} / \mathfrak{K})$ may be lifted to a homotopy of Fredholm operators in $M_{l}(\mathfrak{A})([1], 3.4 .6)$.

Acknowledgements. We thank Severino Collier Coutinho, Ruy Exel, Robert Lauter, Sergiu Moroianu, Anton Savin, Thomas Schick and Mariusz Wodzicki for helpful conversations.

This work was done while the first named author was a guest of the Institute of Mathematics of the University of Potsdam. He wishes to express his gratitude to all members of the group Partial Differential Equations and Complex Analysis for their always gentle hospitality. His visit was supported by the Fundação de Amparo à Pesquisa do Estado de São Paulo (Fapesp, Brazil, Processo 00/00451-2), the European research and training network "Geometric Analysis" (Contract HPRN-CT-1999-00118), and the Deutsche Forschungsgemeinschaft (DFG, Germany).

\section{References}

[1] B. Blackadar, K-Theory for Operator Algebras, Cambridge University Press, 1998.

[2] L. Boutet de Monvel, Boundary problems for pseudo-differential operators, Acta Math. 126 (1971), 11-51.

[3] R. Exel, A Fredholm operator approach to Morita equivalence, K-Theory 7 (1993), 285-308.

[4] G. Grubb, Functional Calculus of Pseudodifferential Boundary Problems, 2nd ed., Birkhäuser, 1996.

[5] G. Grubb and G. Geymonat, The essential spectrum of elliptic systems of mixed order, Math. Ann. 227 (1977), 247-276.

[6] L. Hörmander, Pseudo-differential operators and hypoelliptic equations, in: Singular Integrals (Chicago, 1966), Proc. Symp. Pure Math. 10 (1967), 138-183.

[7] J. J. Kohn and L. Nirenberg, An algebra of pseudo-differential operators, Comm. Pure Appl. Math. 18 (1965), 269-305.

[8] S. T. Melo, R. Nest and E. Schrohe, $C^{*}$-structure and K-theory of Boutet de Monvel's algebra, preprint (http://xxx.lanl.gov/abs/math.OA/0110253); to appear in J. Reine Angew. Math.

[9] S. Rempel and B.-W. Schulze, Index Theory of Elliptic Boundary Problems, Akademie Verlag, 1982.

[10] E. Schrohe, Fréchet algebra techniques for boundary value problems on noncompact manifolds: Fredholm criteria and functional calculus via spectral invariance, Math. Nachr. 199 (1999), 145-185. 